\title{
Análise da qualidade de vida no trabalho de colaboradores com diferentes níveis de instrução através de uma análise de correlações
}

\author{
Luciana da Silva Timossia,*, Antonio Carlos de Francisco ${ }^{\mathrm{b}}$, \\ Guataçara dos Santos Juniorc, Antonio Augusto de Paula Xavier ${ }^{\mathrm{d}}$ \\ a,*lucianatimossi@yahoo.com.br, UTFPR, Brasil \\ bacfrancisco@utfpr.edu.br, UTFPR, Brasil \\ 'guata@utfpr.edu.br, UTFPR, Brasil \\ daugustox@utfpr.edu.br, UTFPR, Brasil
}

\begin{abstract}
Resumo
0 objetivo deste estudo foi investigar a qualidade de vida no trabalho (QVT) de colaboradores com diferentes níveis de instrução, utilizando-se de técnicas estatísticas uni e multivariada para a análise dos dados. Foi realizado por meio da aplicação do modelo de QVT proposto por Walton (1973) em 154 indivíduos - 84 homens e 70 mulheres - com diferentes níveis de instrução. Os resultados gerais indicaram que o critério "compensação justa e adequada" (53) apresentou o menor índice de satisfação adotado e "relevância social", o maior escore $(69,1)$. Na análise de variância com $\alpha=0,05$; valor calculado de $F=1,96 ; \mathrm{P}=0,056$, e valor crítico de $F=2,37$, pode-se afirmar que não existe diferença significativa entre as médias de QVT dos nove níveis de instrução analisados. Quanto à primeira análise de correlação, esta obteve coeficiente de correlação igual a $-0,128$, não tendo sido verificada uma correlação significativa entre o valor geral da QVT dos indivíduos e o nível de instrução. Na segunda análise de correlação multivariada, foi verificado que o nível de instrução apresentou correlação significativa apenas com os critérios 1 e 7 de QVT, com valores respectivos de 0,2480 e 0,2849. Conclui-se que os colaboradores podem apresentar melhor ou pior QVT, independentemente de seu grau de instrução, indicando que todos os colaboradores são sensíveis às modificações e melhorias promovidas pela empresa no ambiente laboral.
\end{abstract}

Palavras-chave

Qualidade de vida no trabalho. Correlação. Nível de instrução.

\section{Introdução}

Os procedimentos que as empresas utilizam para investir recursos no desenvolvimento e na manutenção de seu capital humano são de grande importância, já que a atenção e o cuidado com a saúde dos colaboradores podem estar diretamente relacionados ao desempenho da organização. Segundo Tolfo e Piccinini (2001), estudos relacionados à QVT tiveram seu início nos anos 50, quando pesquisadores como Eric Trist e seus colaboradores do Tavistok Institute, Londres, foram indicados como precursores de investigações ligadas à QVT.

Para algumas empresas, os conceitos de satisfação pessoal no trabalho, de qualidade pessoal e de valorização do capital humano passaram a estar associados ao desenvolvimento da qualidade de serviços e produtos. Atentando a isso, algumas organizações optam por investigar alguns fatores relacionados à saúde dos colaboradores, como: estilo de vida, satisfação, qualidade de vida (QV), qualidade de vida no trabalho (QVT), lazer e hábitos cotidianos.

Percebe-se que o foco das avaliações e análises da QVT depende em grande parte da área de interesse para a qual estão direcionadas. Na área produtiva e industrial, a preocupação em avaliar a QVT surgiu recentemente no Brasil. A partir de uma investigação no Banco de Teses da CAPES, foram encontradas dissertações e teses na área de Administração a respeito da QVT a partir de 1989 . E, a partir de 1996, na área de Engenharia de Produção. 
A avaliação da QVT justifica-se para o indivíduo e para a organização de trabalho pela importância de uma gestão da saúde do colaborador com uma visão mais abrangente, valorizando fatores essenciais para se ter uma boa QVT. A partir do momento em que a empresa possui estas informações, pode então melhor direcionar e fundamentar programas de promoção da saúde de colaboradores, proporcionando melhores condições de trabalho ao individuo, oferecendo-lhe a oportunidade de utilizar todo o seu potencial produtivo.

0 principal objetivo deste estudo foi investigar a QVT em colaboradores com diferentes níveis de instrução, utilizando-se de técnicas estatísticas uni e multivariada para a análise dos dados. As avaliações e as análises constituíram um estudo de caso aplicado, exploratório e predominantemente quantitativo, no qual se pretendeu responder a seguinte questão de pesquisa: Existe correlação significativa entre a QVT e o nível de instrução dos colaboradores?

\section{Investigação da QTV aplicada ao ambente produtivo}

As empresas estão inseridas em um processo contínuo de mudança, desenvolvendo estratégias para acompanhar o aumento da complexidade das relações de mercado. Dentro desse panorama, algumas organizações optam por utilizar a QVT como uma destas estratégias e estão dispensando uma maior atenção às questões relacionadas à valorização do capital humano.

Existem duas estratégias diferentes que as organizações podem adotar quando o foco é o colaborador: a de controle e a de comprometimento. $\mathrm{Na}$ estratégia de controle, os colaboradores são vistos como números, como custo, e tem-se que, para desempenharem bem suas atividades, devem ser mandados e controlados. $\mathrm{Na}$ estratégia de comprometimento, parte-se da consideração de que as pessoas são parceiras na produção e que a organização deve investir para obter os melhores resultados para todos (MARCHI, 2004; LIMONGI FRANÇA, 2004). A premissa de QVT geralmente está associada à estratégia de comprometimento, já que busca a compreensão a respeito de situações individuais dos trabalhadores em seus ambientes laborais.

Segundo Limongi-França e Zaima (2002, p. 406), a QVT pode ser entendida como um

[...] conjunto das ações de uma empresa que envolve a implantação de melhorias e inovações gerenciais, tecnológicas e estruturais no ambiente de trabalho. Estas ações acabam por influenciar o colaborador durante o desenvolvimento de suas atividades.

Para Cole et al. (2005, p. 54).

[...] A qualidade de vida no trabalho inclui largos aspectos do ambiente do trabalho que afetam o colaborador em sua saúde e seu desempenho.

É possível perceber, conforme os Autores citados, que, de forma generalizada, a investigação da QVT pode preocupar-se principalmente com a satisfação do colaborador em relação a inúmeros fatores, como: salário; segurança; saúde no trabalho; condições físicas de trabalho que reduzam ao mínimo o risco de doenças ocupacionais e acidentes de trabalho; desenvolvimento das capacidades e múltiplas habilidades; autonomia; informação; crescimento profissional; integração social, e espaço de vida no trabalho e fora dele.

\section{Metodologia}

Este estudo foi realizado por meio da aplicação de um questionário adaptado do modelo de Walton (1973) para avaliação da QVT em 154 colaboradores com diferentes níveis de instrução, profissões e atividades diversas.

Os indivíduos que participaram do estudo foram selecionados de acordo com seu nível de instrução. A Tabela 1 especifica o número de colaboradores participantes segundo seus ramos e/ou atividades.

A amostra consistiu de 154 pessoas, sendo 84 homens e 70 mulheres. De acordo com os dados sociodemográficos, tem-se que a média de idade dos colaboradores é de 37,6 anos para os homens e de 36 anos para as mulheres. Em relação ao tempo de trabalho, os colaboradores homens apresentaram uma média de 8,9 anos de atuação e as mulheres de 6,2 anos de atuação no mercado de trabalho.

Tabela 1. Participantes do estudo e respectivas atividades.

\begin{tabular}{lc}
\hline \multicolumn{1}{c}{ Ramo e/ou atividade } & N \\
\hline $\begin{array}{l}\text { Alunos do curso de mestrado em Engenharia de } \\
\text { Produção da UTFPR }\end{array}$ & 30 \\
$\begin{array}{l}\text { Profissionais do setor de limpeza, manutenção e } \\
\text { vigilância da UTFPR }\end{array}$ & 24 \\
$\begin{array}{l}\text { Profissionais do setor de limpeza, zelador e copeira } \\
\text { de uma organização tributária federal }\end{array}$ & 8 \\
$\begin{array}{l}\text { Servidores de uma organização tributária federal } \\
\text { Profissionais de uma unidade de alimentação } \\
\text { e nutrição, cozinheiras, auxiliares de cozinha e } \\
\text { balconistas }\end{array}$ & 54 \\
Colaboradores do setor de limpeza, manutenção e & 28 \\
vigilância de um condomínio residencial & 10 \\
Total de participantes da pesquisa & \\
\hline Fonte: Autoria própria, (2008).
\end{tabular}




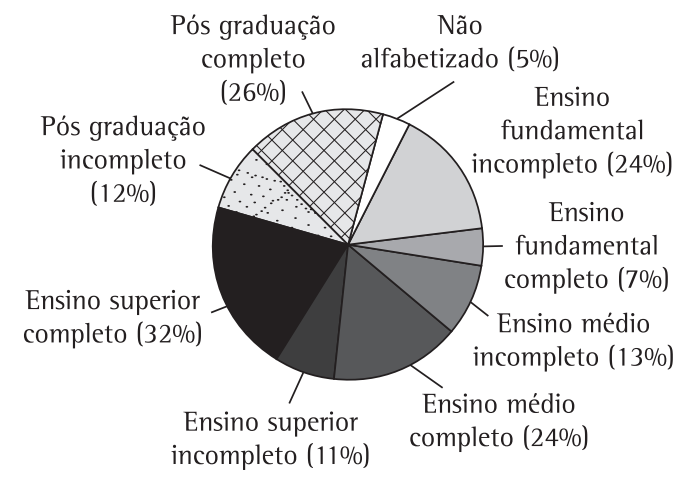

Figura 1. Escolaridade dos respondentes. Fonte: Autoria própria (2008).

Procurou-se utilizar, quanto ao nível de instrução educacional, uma amostra a mais heterogênea possivel, na medida em que é objetivada a sua análise e a correlação desta com a QVT. Assim, a distribuição da população com relação à escolaridade se configura de acordo com a Figura 1.

Com o intuito de possibilitar a análise dos dados, o nível de instrução foi classificado em nove categorias: 1- não alfabetizado (NA); 2- Ensino fundamental incompleto (EFI); 3-Ensino fundamental completo (EFC); 4- Ensino médio incompleto (EMI); 5- Ensino médio completo (EMC); 6- Ensino superior incompleto (ESI); 7- Ensino superior completo (ESC); 8- Pós-graduação incompleta (PGl) e 9- Pós-graduação completa (PGC). Estas nove categorias foram analisadas em conjunto com os dados obtidos a partir da adaptação do instrumento de Walton para a avaliação da QVT.

\subsection{Instrumento de Walton adaptado}

Na avaliação da QVT, foi utilizada uma adaptação do modelo proposto por Walton em 1973. Esta adaptação foi previamente testada e validada em uma amostra de 99 indivíduos, obtendo um alfa de Cronbach de 0,96. 0 instrumento adaptado apresenta os oito critérios propostos por Walton (1973), que se subdividem em 35 questões referentes à QVT e às suas influências sobre o trabalhador. A escolha deste modelo para avaliação da QVT justifica-se porque seus oito critérios abrangem com boa amplitude aspectos básicos das situações de trabalho. E, também, por ser um instrumento flexível, já que os critérios enumerados não estão em ordem de prioridade e podem ser arranjados de maneira distinta para assumir outras importâncias de acordo com a realidade em cada organização. A Tabela 2 indica os critérios presentes no instrumento de QVT, modelo proposto por Walton (1973).
Tabela 2. Critérios de QVT avaliados.

\section{Critério de QVT}

Critério 1. Compensação justa e adequada

Critério 2. Condições de trabalho

Critério 3. Uso e desenvolvimento de capacidades

Critério 4. Oportunidade de crescimento e segurança

Critério 5. Integração social na organização

Critério 6. Constitucionalismo

Critério 7. 0 trabalho e o espaço total de vida

Critério 8. Relevância social do trabalho na vida

Fonte: Adaptado de Walton (1973).

Para as respostas na identificação da percepção dos colaboradores em relação a sua QVT, foi utilizada uma escala do tipo Likert, polarizada em cinco pontos, com os seguintes níveis de satisfação: 1- muito insatisfeito; 2 - insatisfeito; 3 - nem satisfeito nem insatisfeito; 4 - satisfeito, e 5 - muito satisfeito. A proposta desta escala é verificar a satisfação do colaborador em relação ao critério indicado, levando em conta suas necessidades e anseios individuais. Quanto mais alta a pontuação, considerando o valor 5 como máximo para o critério analisado, mais satisfeito está o colaborador.

\section{Análise dos dados: resultados e discussão}

$\mathrm{Na}$ avaliação dos escores obtidos com a aplicação da versão adaptada do modelo de Walton, foram estabelecidos cinco pontos âncoras para as respostas: $0 \% ; 25 \% ; 50 \% ; 75 \%$ e $100 \%$. Estes valores de referência consideram a escala de 0 a 100 para as respostas. Nessa perspectiva, os valores inferiores a $25 \%$ podem ser considerados como predominantemente muito insatisfatórios, enquanto os valores superiores a 75\% caracterizam uma predominância de muita satisfação. Dessa forma, a classificação proposta apresenta a seguinte configuração, descrita na Figura 2.

Como pode ser verificado, somente os critérios com escores acima de 75 foram considerados positivos ou fatores de muita satisfação no ambiente de trabalho. Assim, considerando os valores médios dos oito critérios de QVT, foi verificado que nenhum destes foi avaliado como muito insatisfatório ou muito satisfatório. Analisando as estimativas médias para os oito critérios, pode-se notar que todos os valores encontram-se classificados como satisfatórios.

A segurança quanto a estes resultados, obtidos a partir de índices médios, pode ser maior ainda quando calculado o intervalo de confiança para a estimativa média. 0 cálculo para o intervalo de 
confiança para estimativas médias pode ser efetuado utilizando-se o seguinte procedimento e equação, segundo Triola (2005):

- verificar se as seguintes suposições foram cumpridas: amostra aleatoriamente simples, o $\sigma$ é conhecido, a população parece ser normalmente distribuída ou $\mathrm{n}>30$;

- consultar o valor crítico $Z_{\alpha / 2}$, correspondente ao nível de confiança desejado;

- calcular a margem de erro $E=Z_{\alpha / 2} \sigma / \sqrt{n}$;

Com o valor calculado da margem de erro $E$, juntamente com o valor da média mostral $\mu$, é possível estimar o cálculo do intervalo de confiança para $\mu$ conforme demonstra a Equação 1 (Fonte: Triola (2005, p. 248)):

$$
\bar{x}-Z_{\alpha / 2} \sigma / \sqrt{n}<\mu<\bar{x}+Z_{\alpha / 2} \sigma / \sqrt{n}
$$

A seguir, a Figura 3 apresenta graficamente os intervalos de confiança para os oito critérios, calculados segundo a Equação 1, bem como para a estimativa média geral de QVT com 99\% de confiança.

A visualização acima permite assegurar com uma confiabilidade de 99\% que as verdadeiras médias populacionais para os critérios de QVT encontram-se entre os intervalos representados. Esta apresentação fornece um rigor quanto à apresentação dos resultados, pois quando se trabalha apenas com a estimativa média, ou seja, com amostras e não com a população, não se tem uma noção certa de qual valor pode assumir a verdadeira estimativa média da população.

De acordo com a representação dos intervalos de confiança na Figura 3 e de acordo com a classificação da satisfação em QVT apresentada na Figura 2, todos os critérios apresentaram uma estimativa média superior a 50\%; assim, fica claro que os oito critérios e também o valor médio geral de QVT encontram-se com índices considerados satisfatórios. No critério 1 "compensação justa e adequada" (53), a estimativa média encontra-se na categoria satisfatória; porém, considerando seu intervalo de confiança, existe uma pequena probabilidade do verdadeiro valor da média estar no nível insatisfatório. Este critério apresentou o menor índice de satisfação adotado e, desta forma, pode estar interferindo negativamente na QVT e também indicar relações de conflito entre este fator e o que seria considerado ideal pelos colaboradores.

Os critérios: “oportunidades” $(56,9)$, "condições de trabalho" (61,8), "trabalho e vida" $(62,4)$,

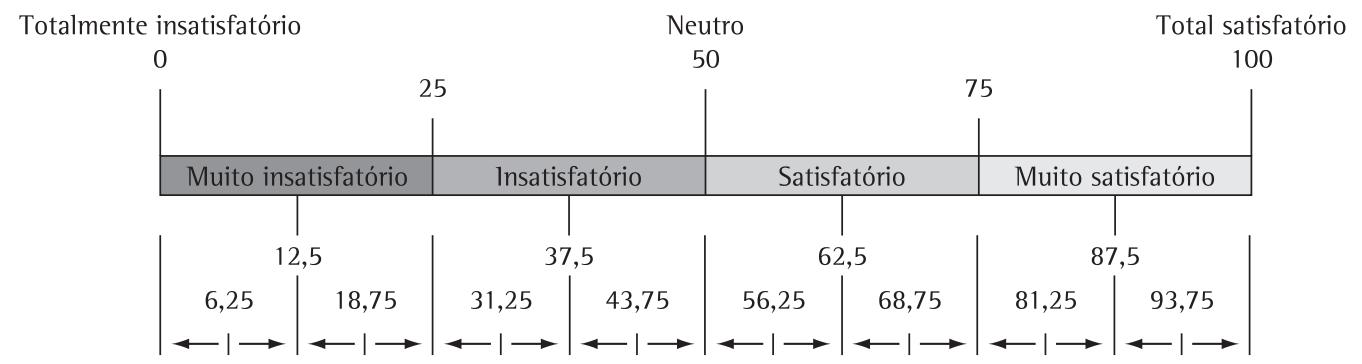

Figura 2. Escala de respostas de QVT utilizada. Fonte: Timossi et al.

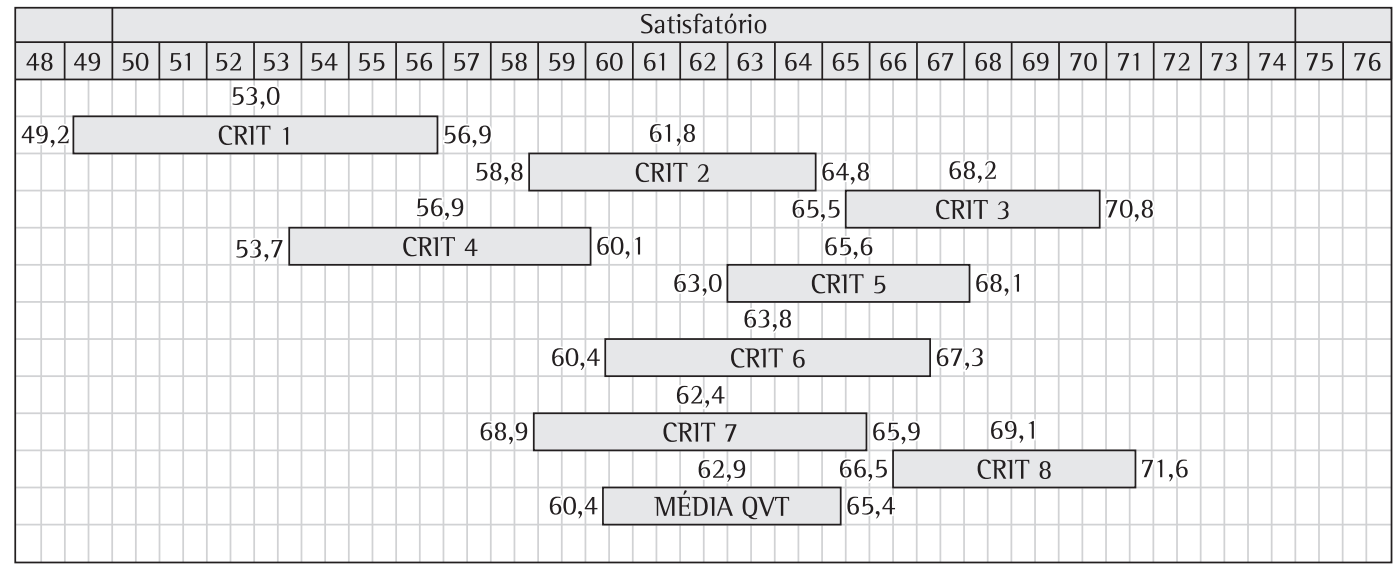

Figura 3. Resultados dos critérios de QVT. Fonte: Autoria própria (2008). 
"constitucionalismo" (63,8), "integração social" $(65,6)$, "uso das capacidades" $(68,2)$, e "relevância social" - com o maior escore $(69,1)$ - apresentaram indices acima de 50\%, indicando satisfação; porém, nenhum dos oito critérios de QVT foi avaliado como muito satisfatório.

Desta forma, o intervalo de confiança pode ser utilizado como uma ferramenta para identificar qual é a tendência da estimativa média com 95\% ou 99\% de confiança, dependendo do critério do pesquisador. Com estes resultados em mãos, é possível orientar a tomada de decisão das empresas na busca por melhores condições laborais para os colaboradores. Lima e Stano (2004) salientam que no atual ambiente competitivo as organizações necessitam de uma força de trabalho saudável, motivada e preparada para a competição, e que as responsabilidades relativas à QVT não são únicas e exclusivas da empresa. Assim, o próprio indivíduo pode ter a consciência de sua importância neste processo ou a organização incentivá-lo ou instruí-lo para tal.

Em continuidade na análise dos dados, foram utilizados outros dois diferentes testes: análise de variância (ANOVA) e análise de correlação (univariada e multivariada), que serão mais bem descritos nos tópicos seguintes.

\subsection{Análise de variância (ANOVA)}

Com o objetivo de verificar se as diferenças entre as estimativas médias de QVT em diferentes níveis de instrução são significativas ou não, aplicou-se o teste de hipótese denominado análise de variância (ANOVA). A análise de variância (ANOVA) é um método para testar a igualdade de duas ou mais médias populacionais, baseado na análise de variâncias amostrais (TRIOLA, 2005). As hipóteses nula e alternativa a serem testadas foram: $H_{0}$ - médias populacionais de QVT para os diversos níveis de instrução são iguais e $H_{1}$ - ao menos uma das médias populacionais de QVT para os diversos níveis de instrução é diferente das demais. Utilizou-se na aplicação do teste de análise de variância um nível de significância igual $\alpha=0,05$, o que representa uma confiabilidade de $95 \%$ nos resultados. Para aplicar a análise de variância, é necessário calcular a estatística de teste $F$, que é composta pela razão de duas estimativas: variância entre amostras e variância dentro de amostras.

Assim, se o valor de $F$ obtido pela equação da estatística de teste for maior que o valor crítico tabelado, referente à estatística teórica, rejeita-se a hipótese nula. Isto indica que existe pelo menos uma diferença significativa entre as médias que estão sendo comparadas. A rejeição ou não da hipótese nula também pode ser verificada por meio do calculo de P. Se o valor $\mathrm{P}$ for menor ou igual a $\alpha=0,05$, rejeita-se a hipótese nula. Se o valor $\mathrm{P}$ for maior que $\alpha=0,05$ não se rejeita a hipótese nula, não havendo, assim, evidências suficientes para afirmar que existem diferenças significativas entre as médias analisadas. A Tabela 3 apresenta os resultados obtidos com a análise de variância ANOVA.

Os resultados gerais indicaram o valor calculado pela estatística $\mathrm{F}=1,96$, e $\mathrm{P}=0,056$, sendo que 0 valor crítico tabela de $F=2,37$. Desta forma, como o valor $F$ calculado é menor que o $F$ crítico, logo não se rejeita a hipótese nula. Portanto, não há evidência suficiente para afirmar que existe pelo menos uma diferença significativa entre as médias de QVT. Desta forma, é possível afirmar que as nove médias de QVT não possuem diferenças significativas entre si, não importando a qual nível de instrução o colaborador pertence. A Figura 4 contém um gráfico boxplot, no qual é possível verificar a dispersão dos dados de QVT para os nove diferentes níveis de instrução e os pontos de interseção dos mesmos. Segundo Triola (2005), o boxplot pode ser usado para descrever as características mais proeminentes de conjuntos de dados. Essas

Tabela 3. Resultados da análise de variância ANOVA.

\begin{tabular}{lcl}
\hline \multicolumn{1}{c}{ Níveis de instrução analisados } & $\mathbf{n}$ & $\begin{array}{c}\text { Média } \\
\text { QVT }\end{array}$ \\
\hline 1- Não alfabetizado & 5 & 74,5 \\
2- Ensino fundamental incompleto & 24 & 69,8 \\
3- Ensino fundamental completo & 7 & 57 \\
4- Ensino médio incompleto & 13 & 61,3 \\
5- Ensino médio completo & 24 & 61,8 \\
6- Ensino superior incompleto & 11 & 57 \\
7- Ensino superior completo & 32 & 57,5 \\
8- Pós-graduação incompleta & 12 & 61,5 \\
9- Pós-graduação completa & 26 & 65,8 \\
Valor de F = 1,96 & & \\
Valor de P = 0,056 & & \\
\hline
\end{tabular}

Fonte: Autoria própria, 2008.

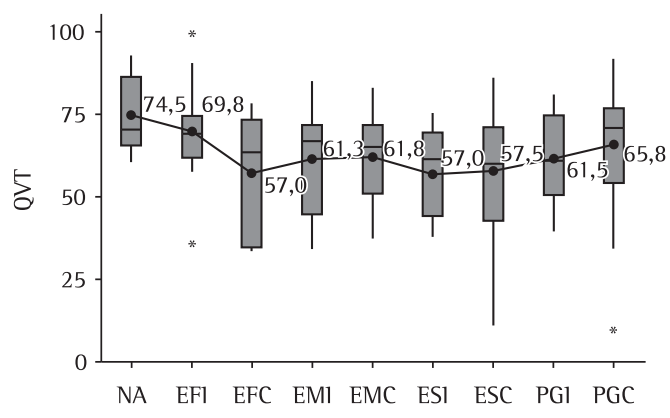

Figura 4. Dispersão dos dados de QVT e seus valores médios para cada nível de instrução. Fonte: Autoria própria, (2008). 
características incluem (1) centro, (2) dispersão, (3) a extensão e a natureza de qualquer desvio em relação à simetria e (4) a identificação de outliers.

Os dados indicam que a média de QVT para o grupo de colaboradores não alfabetizados (NA) - QVT = 74,5 - é maior que a média dos outros grupos. Porém, esta diferença não é significativa. Em sequência, observam-se os seguintes valores: os colaboradores com ensino fundamental incompleto $(\mathrm{EFI})-\mathrm{QVT}=69,8$; com pós-graduação completa (PGC) - QVT $=65,8$; com ensino médio completo (EMC) - QVT = 61,8; com pós-graduação incompleta (PGl) - QVT $=61,5$; com ensino médio incompleto (EMI) - QVT $=61,3$; com ensino superior completo (ESC) - QVT $=57,5$ e, por fim, os colaboradores com menor índice de satisfação no trabalho, com ensino fundamental completo (EFC) e ensino superior incompleto (ESI) - QVT $=57$.

Possivelmente, os fatores e critérios que exercem uma maior representatividade e são mais valorizados dentro da QVT modificam-se de acordo com os níveis de instrução dos colaboradores. $\mathrm{Na}$ medida em que esta sistematização é individual e intrínseca, os fatores que representam satisfação para um determinado grupo de colaboradores podem não representar a mesma fonte de motivação para outros. Logo, neste caso, as organizações de trabalho, às quais estes colaboradores pertencem, parecem estar atendendo melhor as expectativas das pessoas com menor grau de instrução, já que estes colaboradores foram os que apresentaram as duas maiores estimativas médias de QVT.

Walton (1973) previu esta sistematização dos fatores que representam mais ou menos satisfação para determinados grupos de colaboradores quando permitiu a flexibilização do instrumento por ele proposto. O Walton (1973) ainda estabelece que, dependendo do grupo de trabalhadores ou do ambiente, podem ser gerados conjuntos distintos de critérios de QVT que, de acordo com o contexto, podem assumir novas hierarquias de importância na QVT.

Identificou-se também a presença de três outliers no conjunto de dados. Os dados dos colaboradores com ensino fundamental incompleto apresentam dois outliers. um superior e outro inferior. E, nos valores dos colaboradores com pós graduação completa, encontra-se um outlier inferior. De acordo com Triola (2005), um outlier ou um valor discrepante é um valor que se localiza muito distante de quase todos os outros valores, ou seja, é um valor extremo. A exploração de um conjunto de dados pode levar em consideração os outliers, pois estes podem revelar importantes informações e podem afetar muito os valores da média e do desvio-padrão. Assim, os outliers superiores podem ser considerados como colaboradores que possuem um escore realmente muito acima dos demais para aqueles determinados critérios de QVT. Já os outliers inferiores podem ser entendidos como indivíduos que possuem valores muito abaixo dos valores grupais para aqueles critérios.

Salienta-se que a importância da identificação dos outliers na QVT se dá pela possibilidade de identificação dos fatores que levaram aquele colaborador a ter um valor discrepante na QVT; ou seja: permite ao investigador ou ao gestor na empresa verificar o porquê daquele colaborador apresentar valores muito superiores ou muito inferiores para determinado critério.

Portanto, como foi verificada uma igualdade estatística entre as médias de QVT provenientes dos nove diferentes níveis de instrução, optou-se por analisar a correlação (univariada e multivariada) para tentar detectar se existe correlação entre o nível de instrução e o valor de QVT indicado.

\subsection{Análise de correlação multivariada}

Comumente, nos estudos que envolvem a QVT, não se faz uso de técnicas estatísticas multivariadas no tratamento e na análise dos dados. Como exemplo, pode-se observar os estudos realizados por Schmidt (2004) e Trevisan (2005).

\section{Segundo Mingoti (2007, p. 21)}

[...] a Estatística Multivariada consiste em um conjunto de métodos estatísticos utilizados em situações nas quais várias variáveis são avaliadas simultaneamente.

Esta técnica de análise multivariada se faz necessária em avaliações de QVT pela seguinte razão: quando as variáveis em estudo são analisadas isoladamente, perdem-se muitas informações a respeito do fenômeno de estudo, principalmente quando se trata de análise de correlações, em que a univariada, comumente utilizada para se investigar uma possível correlação, não parece ser a melhor alternativa.

É observado que na análise correlação univariada - pelo fato de não se levarem em consideração todas as variáveis envolvidas ao mesmo tempo para se tomar uma conclusão - não se consegue otimizar a extração de informações e é possível que se obtenham conclusões errôneas ou ao menos não tão completas. Para tanto, na verificação de possíveis correlações significativas entre a QVT e outros fatores - neste caso, o nível de instrução -, é necessário utilizar um método que contemple todas as variáveis simultaneamente, ou seja, um método estatístico mais consistente. 
Foram realizadas duas análises distintas. $\mathrm{Na}$ primeira análise, foram comparadas duas variáveis: o nível de instrução dos indivíduos, composto pelas nove possibilidades de escores - 1- (NA); 2- (EFI); 3- (EFC); 4- (EMI); 5- (EMC); 6- (ESI); 7- (ESC); 8- (PGl) e 9- (PGC) - e o valor médio geral de QVT, obtido para cada colaborador. A Figura 5 traz o esquema gráfico da análise.

$\mathrm{Na}$ primeira análise de correlação univariada, obteve-se coeficiente de correlação linear igual a $-0,128$. Assim, considerando a classificação do valor (r) proposta por Munro (2001), percebe-se que os valores (r) encontrados nesta análise encontram-se no intervalo de 0 a 0,25 , o que corresponde a pouca correlação entre as variáveis. Mesmo considerando o método exposto por Triola (2005), não se rejeita a hipótese de correlação não significativa. Como não foi obtida uma correlação significativa entre o valor geral da QVT dos colaboradores e o nível de instrução utilizando uma análise com duas variáveis apenas, optou-se por utilizar uma segunda estratégia.

Desta forma, buscou-se, utilizando agora a análise de correlação multivariada, verificar a existência de correlação significativa entre todos os níveis de instrução dos colaboradores e todos os critérios de QVT. Neste caso, o teste de hipótese sugerido foi o de Bartlett (apud MINGOTI, 2007), formulado conforme a Equação 2:

$T=-\left[n-\frac{1}{6}(2 p+11)\right]\left[\sum_{j=1}^{p} \ln \left(\lambda_{i}\right)\right]$

onde:

- $\mathrm{p}$ : número de variáveis envolvidas no estudo;

- $\lambda$ : autovalores ou valores próprios da matriz de correlação amostral.

Nesta análise, foi correlacionado separadamente o escore referente ao nível de instrução com os oito critérios de QVT propostos por Walton (1973): compensação justa e adequada, condições de trabalho, uso e desenvolvimento de capacidades, oportunidade de crescimento e segurança, integração social na organização, constitucionalismo, o trabalho e o espaço total de vida, e relevância social do trabalho na vida (Tabela 2). Neste caso, ao todo, a análise envolveu nove variáveis: uma referente ao nível geral de instrução e as outras oito referentes aos valores correspondentes aos oito critérios de QVT. A Figura 6 traz o esquema gráfico da segunda análise de correlação.

Considerando que foram avaliados 154 colaboradores $(\mathrm{n}=154)$, os dados analisados constituíram uma matriz de dados com dimensão $154 \times 9$. Desta maneira, é possível construir um teste de hipóteses para a avaliação da matriz de correlação global. Através do cálculo da estatística de teste dada pela Equação 2, obteve-se como resultado o valor $T=878,95$, sendo o valor crítico provindo da distribuição qui-quadrado com nível de significância igual a 0,05 e 36 graus de liberdade igual a 50,99. Como o valor $T=878,95$ obtido para estatística de teste é maior que o valor crítico 50,99, a hipótese nula deixa de ser aceita. Portanto, conclui-se que as nove variáveis em estudo são mutuamente dependentes, ou seja, existe uma correlação significativa entre as mesmas.

Segundo a estatística testada, detectou-se que existe correlação significativa geral entre os nove critérios indicados. Porém, uma importante observação deve ser destacada: ao se detalharem os dados para verificar quais as correlações significativas encontradas, pode ser observado que o valor da correlação geral significativa $T=878,95$ provavelmente originou-se pela correlação entre os oito critérios de QVT com eles próprios, sendo que o fator "nível de instrução" continuou a não

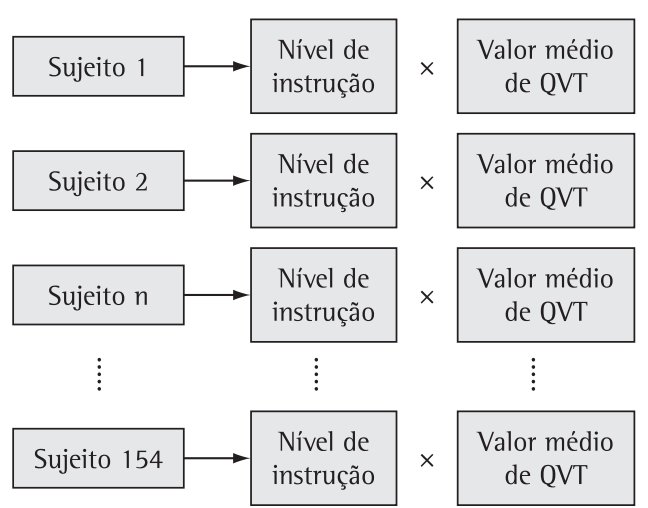

Figura 5. Primeira análise de correlação. Fonte: Autoria própria (2008).

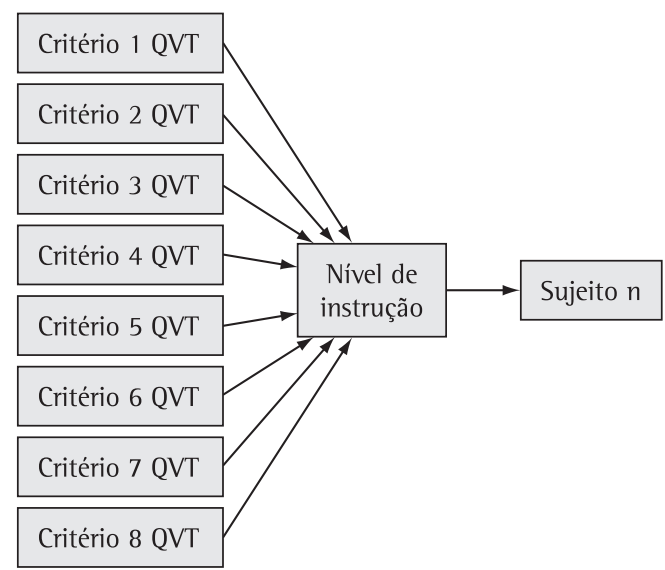

Figura 6. Segunda análise de correlação. Fonte: Autoria própria (2008). 
apresentar correlação com a maioria dos oito critérios da QVT. A seguir, podem ser identificados os valores de correlação significativos tanto dos critérios de QVT entre si quanto do nível de instrução com os critérios de QVT.

Para que se possa afirmar que existe uma correlação significativa, considera-se como valor crítico de correlação para um $\mathrm{n}=154$ e um nível de significância de 0,05 o valor de 0,161. Ou seja: todos os valores apresentados na Tabela 4, que são superiores ao valor crítico de 0,161 , indicam que há uma correlação estatisticamente significativa entre os domínios e os critérios relacionados. Os coeficientes que apresentaram correlação significativa estão destacados em amarelo.

É perceptível que todos os oito critérios de QVT apresentam correlações significativas entre si, sendo o coeficiente 0,7418 o mais alto e o coeficiente 0,4696, o mais baixo. Quanto ao nível de instrução, é notório que este não apresentou correlação significativa com os critérios 2, 3, 4, 5, 6 e 8 da QVT. Os dois critérios nos quais se obteve uma correlação significativa - 1 "compensação justa e adequada" e 7 "trabalho e vida", com valores respectivos de 0,2480 e -0,2849 - apresentaram um coeficiente que, apesar de significativo, também é considerado fraco. 0 indicado, segundo autores, como Munro (2001) e Xavier (2000), seria atingir coeficientes acima de 0,6, considerando que a QVT é uma variável subjetiva, para então representar uma correlação que, além de significativa, seja também forte. Este parâmetro de coeficiente de correlação 0,6 se baseia em estudos que também correlacionaram variáveis subjetivas e opiniões de indivíduos. Xavier (2000) estudou as sensações térmicas relatadas por pessoas e afirma que é muito complexa a obtenção de um modelo analítico para representar opiniões e sensações das pessoas devido às inúmeras possibilidades de influências subjetivas atuantes. Xavier (2000, p. 159) ainda acrescenta que
[...] de maneira geral, pode-se considerar, para um modelo de utilização genérica, que um coeficiente de determinação $R^{2}=0,6$ indica que o mesmo é uma boa ferramenta de avaliações e predições.

Considerando o critério "compensação justa e adequada" vs. "nível de instrução", obteve-se uma correlação significativa. Este achado pode ser assim explicado: quanto maior o nível formal de instrução, maior tende a ser a remuneração do colaborador, pois este se torna mais especializado. Fleck (2008) relatou uma metodologia desenvolvida pelo periódico "The Economist": um índice de qualidade de vida baseado em correlações entre índices de satisfação subjetiva com a vida com fatores determinantes objetivos da qualidade de vida. 0 principal fator associado a uma boa satisfação com a vida foi a renda. Fleck (2008) ainda acrescenta que uma boa situação socioeconômica mostra-se associada a uma boa qualidade de vida. Assim, pode-se aventar que investimentos na instrução dos colaboradores são capazes de levar a um melhor nível de renda, o que, consequentemente, pode proporcionar uma melhor qualidade de vida geral.

A questão remuneração, envolvendo salário e benefícios, pode ser considerada e conhecida através dos estudos da psicologia baseados em Herzberg, os quais compreendem que a remuneração tem uma função higiênica sobre a motivação, sendo que o salário e os benefícios não funcionam como fonte de motivação e sim evitam a desmotivação (TOLFO; PICCININI, 2001).

0 critério "trabalho e vida" vs. "nível de instrução" apresentou uma correlação negativa -0,2849. Entande-se, assim, que quanto maior o nível de instrução, menos satisfeito estará o colaborador quanto ao espaço do trabalho em sua vida pessoal. Este achado justifica-se, pois quanto maior o nível de instrução, aumentam-se também as chances de assumir maiores responsabilidades no trabalho, o

Tabela 4. Coeficientes de correlação dos critérios de QVT e do nível de instrução.

\begin{tabular}{|c|c|c|c|c|c|c|c|c|c|}
\hline Crit 1 & Crit 1 & & & & & & & & \\
\hline Crit 2 & 0,67 & Crit 2 & & & & & & & \\
\hline Crit 3 & 0,47 & 0,66 & Crit 3 & & & & & & \\
\hline Crit 4 & 0,58 & 0,74 & 0,71 & Crit 4 & & & & & \\
\hline Crit 5 & 0,47 & 0,58 & 0,61 & 0,59 & Crit 5 & & & & \\
\hline Crit 6 & 0,59 & 0,70 & 0,64 & 0,72 & 0,74 & Crit 6 & & & \\
\hline Crit 7 & 0,61 & 0,64 & 0,56 & 0,62 & 0,55 & 0,65 & Crit 7 & & \\
\hline Crit 8 & 0,49 & 0,60 & 0,67 & 0,65 & 0,64 & 0,69 & 0,5462 & Crit 8 & \\
\hline $\begin{array}{c}\text { Nível } \\
\text { instrução }\end{array}$ & 0,25 & $-0,10$ & $-0,06$ & $-0,02$ & 0,03 & $-0,09$ & $-0,2849$ & 0,03 & $\begin{array}{c}\text { Nível } \\
\text { instrução }\end{array}$ \\
\hline
\end{tabular}

Fonte: Autoria própria (2008). 
que reflete em mais tempo dedicado às atividades laborais. Assim, pode surgir um desequilíbrio entre o tempo dedicado ao trabalho e o tempo fora deste. Vasconcelos (2008) descreveu estudos envolvendo mais de mil executivos brasileiros e relatou que o fato de subir na carreira é prejudicial à saúde tanto de homens como de mulheres, e que 54\% destes executivos estão insatisfeitos com o pouco tempo dedicado à vida pessoal. Desta forma, pode-se pensar que a falta de equilíbrio entre atividades profissionais e a vida pessoal pode levar a um desgaste crescente dos colaboradores.

Contudo, isto não quer dizer que investir na qualificação dos colaboradores é negativo; o interessante seria investir em uma melhor forma de gerir o tempo, buscando um equilíbrio entre tempo de trabalho e tempo para atividades pessoais. Segundo Tolfo e Piccinini (2001), a questão da qualificação dos colaboradores vem sendo amplamente debatida no meio acadêmico e profissional, e coloca-se como ponto central à frente da Terceira Revolução Industrial e da era do conhecimento. Acrescentam ainda que, no Brasil, esta temática é especialmente preocupante, pois a média de instrução formal dos brasileiros é de quatro anos, enquanto a média dos argentinos é de nove anos e a dos chineses, de 13 anos.

Em um estudo realizado por Rodrigues (1999), utilizando o modelo de Hackman e Oldham para avaliar a QVT, foram encontrados resultados semelhantes quanto a não correlação da QVT e com o nível de instrução. Nesta pesquisa, o Autor avaliou 18 empresas do ramo de confecções do Estado do Ceará, em 1994. 0 trabalho atingiu um total de 104 executivos e investigou a QVT, a motivação e a satisfação dos profissionais. As principais conclusões deste trabalho dizem respeito à motivação alta para o trabalho, influenciada pelo nível de instrução formal - quanto maior o nível de instrução formal, maior a motivação - e pelo estado civil: os solteiros apresentaram baixa motivação; casados, motivação mediana, e desquitados/divorciados, uma alta motivação. Por sua vez, a satisfação com o trabalho apresentou-se inversamente proporcional ao nível de instrução formal e diretamente proporcional em relação ao nível salarial.

De forma geral, os resultados obtidos na análise de correlação univariada e multivariada indicam que aspectos relevantes, como os níveis de instrução e a QVT, agora podem ser discutidos de maneira mais fundamentada e segura. Estes resultados mostram que o valor de QVT, tanto analisado de maneira geral como analisado por critérios, não apresenta correlação linear com o nível de instrução formal dos indivíduos, permitindo aos colaboradores apresentar melhor ou pior QVT, independentemente de seu grau de instrução. Para as empresas, este pode ser um importante indicador, pois mostra que todos os colaboradores, não importando seu nível de instrução, estão sensíveis às modificações, implantações e melhorias promovidas no ambiente laboral.

\section{Conclusão}

Este estudo, no intuito de investigar a QVT em colaboradores com diferentes níveis de instrução, através de técnicas estatísticas uni e multivariada, obteve as seguintes conclusões.

Os dados de dispersão indicam que a população não alfabetizada apresenta a maior média de QVT $=74,5$; com menores índices de satisfação no trabalho, estão os colaboradores com ensino fundamental completo (EFC) e ensino superior incompleto (ESI), ambos com QVT $=57$. Porém, o teste de análise de variância ANOVA indicou que esta diferença não é significativa, não existindo diferença estatística entre as estimativas médias referentes aos nove níveis de instrução analisados.

$\mathrm{Na}$ primeira análise de correlação univariada, obteve-se um coeficiente de correlação linear igual a $-0,128$, indicando que nesta forma de análise, não foi obtida uma correlação significativa entre o valor geral da QVT com o nível de instrução. Na segunda análise de correlação multivariada, o valor $T=878,9531$ obtido para estatística de teste é maior que o valor crítico 50,9985; portanto, conclui-se que existe uma correlação significativa entre as variáveis em estudo. Porém, ao detalharem-se os dados, verificou-se que as correlações significativas encontradas se referem aos oito critérios de QVT com eles próprios. 0 nível de instrução apresentou correlação significativa apenas com os critérios 1 "compensação justa e adequada" e 7 "trabalho e vida”, de QVT com valores respectivos de 0,2480 e -0,2849, o que indica um coeficiente, apesar de significativo, também fraco.

Estes resultados mostram que a QVT, tanto analisada de maneira geral como analisada por critérios, não apresenta correlação linear suficientemente significativa e forte com o nível de instrução dos indivíduos. Conclui-se que os colaboradores podem apresentar melhor ou pior QVT, independentemente do seu grau de instrução. Isso também indica que todos os colaboradores são sensíveis às modificações, implantações e melhorias promovidas pela empresa no ambiente laboral, não importando o seu nível de instrução para indicar uma QVT com tendência positiva ou negativa. 


\section{Referências}

COLE, D. C. et al. Quality of working life indicators in Canadian health care organizations: a tool for healthy, health care workplaces? Occupational Medicine, v. 55, n. 1, p. 54-59, 2005.

FLECK, M. P. A. et al. A avaliação da qualidade de vida: guia para profissionais da saúde. Porto Alegre: Artmed, 2008.

LIMA, W. D.; STANO, R. C. T. M. Pesquisa de clima organizacional como ferramenta estratégica de gestão da qualidade de vida no trabalho. In: SIMPÓSIO DE ENGENHARIA DE PRODUÇÃO, 11., 2004, Bauru. Anais... Bauru: UNESP, 2004. p. 1-8.

LIMONGI-FRANÇA, A. C. Qualidade de Vida no Trabalho: QVT. 2. ed. São Paulo: Atlas, 2004.

LIMONGI-FRANÇA, A. C.; ZAIMA, G. Gestão da qualidade de vida no trabalho - GQVT. In: MANUAL de gestão de pessoas e equipes: estratégias e tendências. São Paulo: Gente, 2002. p. 78-87.

MARCHI, D. Saúde-nutrição do trabalhador num contexto de mudança organizacional. 2004. 185 f. Tese (Doutorado em Engenharia de Produção) - Universidade Federal de Santa Cataria, Florianópolis, 2004.

MINGOTI, S. A. Análise de dados através de métodos de estatística multivariada: uma abordagem aplicada. Belo Horizonte: Editora UFMG, 2007.

MUNRO, B. H. Statisticals methods for health care research. 4. ed. Philadelphia: Lippincott, 2001.

RODRIGUES, M. V. C. Qualidade de vida no trabalho: evolução e análise no nível gerencial. 6. ed. Petrópolis: Vozes, 1999.

SCHMIDT, D. R. C. Qualidade de vida e qualidade de vida no trabalho de profissionais de enfermagem. 2004. 197 f. Dissertação (Mestrado em Enfermagem) - Escola de Enfermagem de Ribeirão Preto, Universidade de São Paulo, Ribeirão Preto, 2004.
TIMOSSI, L. S. et al. Evaluation of quality of work life: an adaptation from the Walton's OWL model. In: INTERNATIONAL CONFERENCE ON INDUSTRIAL ENGINEERING AND OPERATIONS MANAGEMENT ICIEOM, 14., 2008. Rio de Janeiro: ABREPO, 2008. p. 1-13.

TOLFO, S. R.; PICCININI, V. C. As melhores empresas para trabalhar no Brasil e a qualidade de vida no trabalho: disjunções entre a teoria e a prática. $R A C$, v. 5 , n. 1, p. 165-193, 2001.

TREVISAN, E. M. B. M. A dinâmica satisfação-sofrimento e a qualidade de vida no trabalho de uma equipe de saúde no atendimento à criança queimada. 2005. $165 \mathrm{f}$. Tese (Doutorado em Engenharia de Produção) - Universidade Federal de Santa Catarina, Florianópolis, 2005.

TRIOLA, M. F. Introdução à estatística. 9. ed. Rio de Janeiro: LTC, 2005.

VASCONCELOS, A. F. Espiritualidade no ambiente de trabalho. São Paulo: Atlas, 2008.

WALTON, R. E. Quality of working life: what is it? Slow Management Review, v. 15, n. 1, p. 11-21, 1973.

XAVIER, A. A. P. Predição de conforto térmico em ambientes internos com atividades sedentárias - teoria física aliada a estudos de campo. Florianópolis, 2000. 254 f. Tese (Doutorado em Engenharia de Produção) - Universidade Federal de Santa Catarina, Florianópolis, 2000.

\section{Agradecimentos}

Os autores agradecem pelo apoio concedido pela Coordenação de Aperfeiçoamento de Pessoal de Nível Superior (CAPES) e pelo Conselho Nacional de Desenvolvimento Científico e Tecnológico (CNPq), através do suporte financeiro recebido para a elaboração da pesquisa.

\title{
Analysis on quality of work life of employees with different levels of education through an analysis of correlations
}

\begin{abstract}
The objective of this study was to investigate the quality of working life (QWL) in workers with different levels of education, through the use of uni- and multivariate statistical techniques to analyze the data. This study was carried out through the application of a QWL model proposed by Walton (1975) on 154 individuals (84 men and 70 women), with different educational levels. The general results indicated that the criterion "fair and adjusted compensation" (3.1) showed a lower dissatisfaction index and the criterion "social relevance" the lowest (3.74). In the analysis of variance with $P=0,05$; calculated value of $F=1,96 ; P=$ 0,056 and critical value of $F=2,37$, it is possible to affirm that there is no significant difference in the QWL mean among the 9 analyzed levels of instruction. Concerning the correlation analysis, the first analysis obtained a correlation value equal to -0.128 , where a significant correlation between the QWL and the educational level of the individuals was not found. In the second correlation analysis, it was confirmed that the instruction level showed significant correlation only with criteria 1 and 7 of QWL with respective values of 0.2480 and 0.2849 . It is possible to conclude that the collaborators may manifest a better or worse QWL, independently of their level of education. This also indicates that all the collaborators are sensitive to the modifications and innovations and improvements promoted by the company in the labor environment.
\end{abstract}

Keywords

Quality of working life. Correlation. Education degree. 